\title{
RISK ASSESSMENT OF ZOONOTIC CUTANEOUS LEISHMANIASIS CASES AMONG MANUAL WORKERS IN EGYPT
}

\author{
By \\ AHMED MEGAHED AHMED SALEH ${ }^{1}$, NARGIS ALBERT LABIB ${ }^{2}$, \\ MOHAMED BAKR EL-ATTAR ${ }^{1}$ AND TOSSON A. MORSY ${ }^{3}$
}

Military Medical Academy ${ }^{1}$, Cairo 11291, Department of Public Health ${ }^{2}$, Faculty of Medicine, Cairo University and Department of Parasitology ${ }^{3}$, Faculty of Medicine, Ain Shams University, Cairo 11566, Egypt

Abstract

The Egyptian endemic foci of cutaneous leishmamasis were mainly in the Sinai Peninsula. To study the epidemiological characteristics of zoonotic cutaneous leishmaniasis cases in special groups in endemic area in Sinai, a descriptive study was conducted to assess the risk of cutaneous leishmania within spreading places in Sinai among special groups working in endemic areas, during the study period from January- December 2015. All patients in the study setting described before were submitted to clinical examination and history taking based on epidemiologically linked diagnosis. Their total number was (124) cases divided into (85) patients locally treated and (39) patients referred to central hospital.

The results showed that $(85.5 \%)$ was in middle education class, had stable work (87\%) and working in manual services (91.1). A total of 58.1\% slept outdoor at the dusk time (54\%). Three quarters $(75.8 \%)$ didn't use insect repellant and 56.55 didn't use bed nets. Sandy ground, mountain and desert surrounds geographic environment $(100 \%, 97.6 \%$ \& 96\%) respectively. They lived in one floor buildings (85\%), dormitories habitat $(98 \%)$, soiled $(92 \%)$, have cracks on the wall $(60 \%)$, ceiling made of wood $(74 \%)$ and fence was not available in majority of buildings $(96.8 \%)$. Disfiguring was commonest complain in 55\% of cases. The lesions $(85 \%)$ were ulcers $(2 \times 2 \mathrm{~cm}$ in size $(51 \%)$, at lower limb (55\%) and two in numbers in $(50 \%)$. All cases were treated with fluconazole and $8.9 \%$ was cured. Stibogluconate was given to $91 \%$ and with cure rate of $53 \%$. Both Cryotherapy and laser therapy was applied to $16 \%$ of cases. Treatment cured $87 \%$ but $39.5 \%$ suffered scar complications with main sequelae $(15.3 \%)$.

Key words: Egypt, ZCL, Workers, Geographic factors, Ulcers, Cryotherapy, Stibogluconate

\section{Introduction}

Leishmaniasis infection was listed by the WHO among the six most important tropical diseases, being endemic in many different countries worldwide with global estimate of 350 Million individuals at risk (WHO, 2013). CDC (2004) reported more than 12 million chronic cases worldwide. But, there was an increased incidence partially attributed to geographical expansion, population migration, international travel and/or deployment of military forces. Zoonotic cutaneous leishmaniasis (ZCL) was endemic in almost all the Eastern Mediterranean countries countries (Postigo, 2010) as well as in the nearby countries as Jordan (Morsy and el Ajlouny, 1984), Lybia (Fathy et al, 2009), Saudi Arabia (Mohammadi and Alhussainy, 2014), Palestine (Al-Jawabreh et al, 2016), Israel (Ben-Shimol et al, 2016), and Sudan
(Karimkhani et al, 2016).

In Egypt, the endemic foci of the zoonotic cutaneous leishmaniasis were reported North and South Sinai and Suez Canal Zone (El Gibali and El Mansouri, 1979; Morsy, 1983; 1996; Bassili et al, 1983; Abdel Wahab et al, 1986; Mansour et al, 1987; Faris et al, 1986, 1988; Amer and Morsy, 1995: Hamadto et al, 2003; 2007; Morsy, 1996; 2012; 2013; Morsy et al, 1995; 1997). Leishmania major was the Culprit parasite (Wahba et al, 1990; Dawoud, 2004), and Phlebotomus papatasii proved to the main vector (Merdan et al, 1992; Fahmy et al, 2009). Hanafi et al. (2001) reported Ph. sergenti the vector of $L$. tropica as a new record in the southern Nile valley and Shehata et al. (2009) reported the first case of $L$. tropica from a classical focus of L. major in North Sinai Governorate.

As to ZCL reservoir(s), Morsy et al. (1996) 
reported that in the Middle East, the fat rat Psammomys obesus is the most important reservoir animal of ZCL were identified in Sinai, Meriones sacramenti (Morsy et al. (1993) and Gerbillus pyramidum (Morsy et al. (1994) as well as the red fox Vulpes $O$. aegyptica is a new reservoir host (Morsy et al, 2002).

Rationale and significance: Based on the information recorded and noticed, there was an increased referral and admission of cutaneous leishmanial cases, in a general military hospital, so it was necessary to study the ecological factors for spread of this disease, Hence assessing the situation of the problem and diminishing the burden of the disease on the affected at risk group.

The work aimed to minimize or even control the spread of zoonotic cutaneous leishmaniasis in the spreading places in Egypt especially among the manual workers.

Objectives: 1- To study the prevalence of cutaneous leishmaniasis cases among manual workers along the year of 2015. 2- To identify the ecological factors favoring its spread. 3- To study the socio-demographic characteristics and predisposing factors serving as a risk factors for distribution of cutaneous leishmaniasis. 4- To describe the lines of proper prevention and management of that disease. 5- To know the potential variables that might set leishmaniasis hazards to be scattered all over Egypt and its neighboring countries and vice versa. 6- To set recommendations that should be followed for prevention of the disease.

\section{Subjects and Methods}

The study assessed the risk of cutaneous leishmania transmission within spreading places in Egypt aiming to minimize or even control its spread especially among the manual workers.

Technical design: This design entails description of the research design, setting subjects and data collection tools.

Study Design: A descriptive study design was conducted to achieve the aim of the study among the manual workers, over the period of one year.

Study setting: The study was conducted in North Sinai Governorate located in the northeastern part of Egypt $\left(30.5^{\circ} \mathrm{N} 33.6^{\circ} \mathrm{E}\right)$ marking the point of connection between Asia and Africa. North Sinai is bordered by Gulf of Suez, Red Sea and Mediterranean Sea and is habited mainly by Bedouins. The regions comprise the following districts: ElHassan, Beer El-Abd, Nekhel, Sheikh Zuuweid, Beer lehfen and Rafah. Beer Lehfen District was selected to conduct the study, based on its central location in North Sinai Governorate, the convenient communication and distribution of ZCL cases to understand the potential role of both sand-fly vector and rodents-reservoirs in the dynamics of zoonotic leishmaniasis transmission. This district has the diverse geographic and demographic characteristics and its "Crossroads" nature and environmental changes may create the new potential risks for disease transmission. The weather in North Sinai is characterized as hot and dry with marked differences in temperature between day and night. Dramatic weather-related changes as presented by the annual averages of environments factors during the study period from January to December 2015, as reported by the majority of patients.

All patients recruited in the study setting were included. All cases were locally submitted to clinical examination and history taken based on epidemiologically linked diagnosis, their total number was (124) cases divided into (85) patients locally treated and (39) patients referred to central hospital for treatment by the dermatologist.

Inclusion criteria were personnel with distinct skin lesion, and new arrival to Sinai.

Exclusion criteria were 1- Bedouin, 2Subjects refuse to share in the study or confused skin lesion, could be followed-up, or with tiny small cutaneous lesion.

Data collection tools: Two tool of data collection ware adopted: I. Structured questionnaire: identifying the ecological factors enhancing leishmaniasis spread, this tool con- 
sisted of two parts. First part: it includes demographic data as (age, sex, rank, occupation, education level, stay length). Second part: it includes the description of living and working environment in the study setting such as (land, building, and vegetation, and water collection, presence of insect-vector and animal-reservoir). 2. Patient sheet: finding out the epidemiology of the intended disease and consisted of: First part: included history of present illness (main complaint, lesion discovery, diagnosis, treatment, and outcome. Second part: included characteristics of skin lesion such as (type, size, site, number, complication, management of referral.

II. Operational design: 1- Pilot study to examine the clarity of language and feasibility of questionnaire validation tool and its relevance to study. It also helped to estimate the time needed for filling the questionnaire. A pilot study was conducted including $10 \%$ of cases. A final version of the tool was achieved after doing modification, addition and or omission of item of the tool. The result of the pilot study was not included in the main study data. 2- Field work: covered 12 months duration. It started from the first of January to the end of December 2015and extended to May 2016. Data were collected by personal interview by fulfilling structured questionnaire after being pre-tested in pilot study. Data were collected by well-trained interviewers under close supervision of the authors who visited the setting once every month because of security challenges. However, some data were recalled by phone.

Ill. Administrative design: The study protocol was reviewed and approved by the Committee of Military Institute of Health and Epidemiology. Once approval, an official formal letter was obtained. The aim of the study was explained to the director of the hospital, doctor and patients to obtain their written consent to conduct the study.

Ethical consideration: The permission and official approvals to carry out the study was obtained from the director of the Military Hospital for data collection. Consent was taken from the subjects of the study before the recruitment in the study sample. The questionnaire form was anonymous and only codes were used for precisions of data collection from subjects. All collected data was strictly confidential and wasn't disclosed for any reason but used only for study purposes.

Statistical analysis: Data were revised and entered into Microsoft Excel 2010 and exported to be analyzed using IBM SPSS for Windows, Version 21.0. Armonk, NY: IBM Corp. Data was summarized frequency and percentage for qualitative variables. Comparison between groups was performed using Chi square or Fisher's exact test for qualitative variables. Logistic regression was done to predict the factors with significant prediction of treatment failure. $\mathrm{P}$ values less than 0.05 were considered significant. Chi-Square test: to test the association between qualitative nominal variables, it was performed mainly on frequencies and determined whether the observed frequencies differ significantly from expected frequencies.

Limitations of study:1- Security threats, 2Confidentiality defect, 3- Outreach to the place of study was very dangerous, 4- Difficulty to get sample from lesion for further diagnosed and 5-Restricting full data to obtain.

Points of weakness: The remote area, large scale of manual workers deployment, working under risky and stressful circums- tances, difficult referral or lack of reporting system.

Points of strengths: Easy to control groups, closed confined community, no attrition, good communication (strategic lines), availability of repeated examinations and follow up, plenty of chemotherapy in site (fluconazole, Na-stibogluconate), Medico-legal consideration (every patient received treatment), or strictly following medical rules (more compliance).

The results were given in tables (1 to 28 ). 
Time of study (Gantt chart)

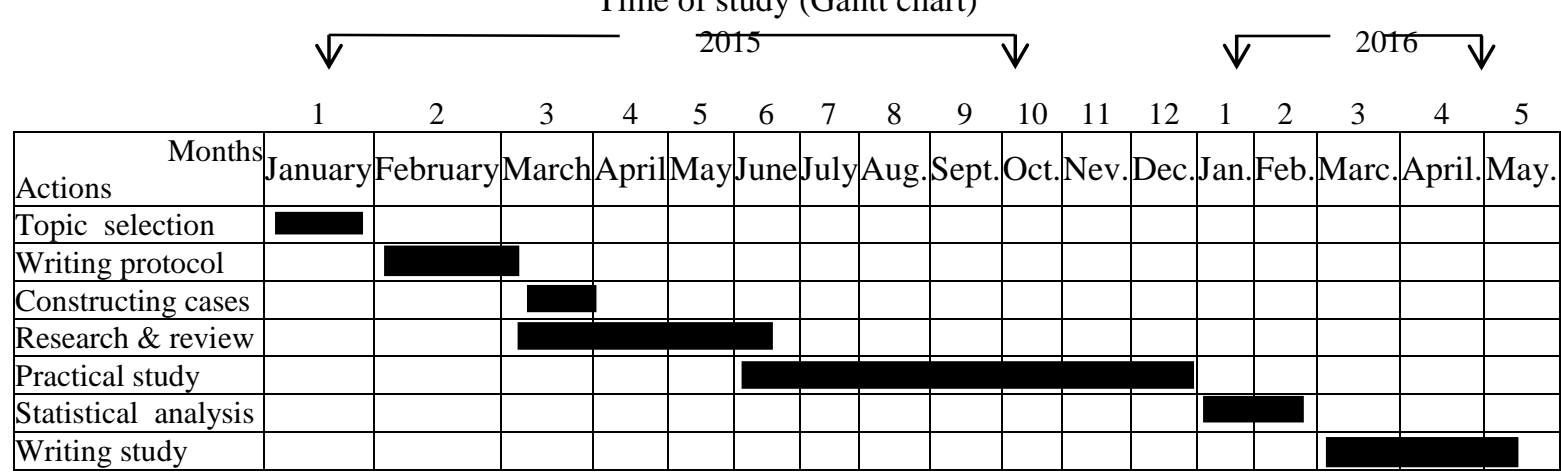

\section{Results}

Table 1: Sociodemographic characteristics of samples

\begin{tabular}{|c|c|c|c|}
\hline Items & Variants & No & Percentage \\
\hline \multirow[t]{2}{*}{ Age } & $20-30$ & 117 & 94.4 \\
\hline & $>30$ & 7 & 5.6 \\
\hline Sex & Male & 124 & 100.0 \\
\hline \multirow{4}{*}{$\begin{array}{l}\text { Previous } \\
\text { Occupation }\end{array}$} & Student & 47 & 37.9 \\
\hline & Farmer & 48 & 38.7 \\
\hline & Laborer & 15 & 12.1 \\
\hline & Administrative worker & 14 & 11.3 \\
\hline \multirow[t]{3}{*}{ Education } & Read \& write & 5 & 4.0 \\
\hline & Middle education & 106 & 85.5 \\
\hline & Higher education & 13 & 10.5 \\
\hline \multirow[t]{4}{*}{ Blood group } & $\mathrm{A}$ & 55 & 44.4 \\
\hline & $\mathrm{AB}$ & 1 & .8 \\
\hline & $\mathrm{B}$ & 29 & 23.4 \\
\hline & $\mathrm{O}$ & 39 & 31.5 \\
\hline \multirow[t]{3}{*}{ Original residence } & Upper Egypt & 76 & 61.3 \\
\hline & Lower Egypt & 33 & 26.6 \\
\hline & Suez Canal Zone & 15 & 12.1 \\
\hline
\end{tabular}

Table 2: Work characteristics of samples

\begin{tabular}{|c|l|c|c|}
\hline & & No. & Percentage \\
\hline \multirow{3}{*}{ Duration (months) } & $<6$ month & 5 & 4.0 \\
\cline { 2 - 4 } & $6-12$ month & 116 & 93.5 \\
\cline { 2 - 4 } & $>12$ month & 3 & 2.4 \\
\hline \multirow{3}{*}{ Nature of work } & stable & 108 & 87.1 \\
\cline { 2 - 4 } & movable & 9 & 7.3 \\
\cline { 2 - 4 } & Variable/mix & 7 & 5.6 \\
\hline \multirow{3}{*}{ Current task } & military services & 113 & 91.1 \\
\cline { 2 - 4 } & logistic services & 9 & 7.3 \\
\cline { 2 - 4 } & deputy & 2 & 1.6 \\
\hline
\end{tabular}

Table 3: Prevention knowledge $\&$ practices/risk taking behavior

\begin{tabular}{|c|l|c|c|}
\hline & & No. & Percentage \\
\hline \multirow{3}{*}{ Endemic information } & No & 113 & 91.1 \\
\cline { 2 - 4 } & Yes & 4 & 3.2 \\
\cline { 2 - 4 } & didn't know & 7 & 5.6 \\
\hline \multirow{3}{*}{ Sleeping pattern } & Outdoor & 72 & 58.1 \\
\cline { 2 - 4 } & Indoor & 24 & 19.4 \\
\cline { 2 - 4 } & Both & 28 & 22.6 \\
\hline \multirow{3}{*}{ Sleeping time } & Dusk & 67 & 54.0 \\
\cline { 2 - 4 } & Mid night & 25 & 20.2 \\
\cline { 2 - 4 } & Dawn & 32 & 25.8 \\
\hline \multirow{2}{*}{ Using insect repellants } & No & 94 & 75.8 \\
\cline { 2 - 4 } & not know & 30 & 24.2 \\
\hline \multirow{2}{*}{ Using bed nets or cover } & No & 70 & 56.5 \\
\cline { 2 - 4 } & Yes & 54 & 43.5 \\
\hline
\end{tabular}


Table 4: Ecological factors of surrounding area of sample

\begin{tabular}{|l|c|c|c|}
\hline \multicolumn{1}{|c}{ Items } & Variants & No. & Percentage \\
\hline \multirow{2}{*}{ Geographical factors } & Mountains & 121 & 97.6 \\
\cline { 2 - 4 } Surrounding area & Hills & 3 & 2.4 \\
\hline Ambient ground & Desert & 119 & 96.0 \\
\hline \multirow{2}{*}{ Presence of vegetation } & Residential & 5 & 4.0 \\
\hline Presence of water collection & sandy & 124 & 100.0 \\
\hline \multirow{3}{*}{ Presence of animal } & desert plant & 114 & 91.9 \\
\cline { 2 - 4 } & trees & 10 & 8.1 \\
\hline \multirow{3}{*}{ Seeing sand fly } & No & 124 & 100.0 \\
\cline { 2 - 4 } & rodent & 108 & 87.1 \\
\cline { 2 - 4 } & dog & 18 & 14.5 \\
\hline \multirow{3}{*}{ Breeding place availability } & cat & 19 & 15.3 \\
\cline { 2 - 4 } & No & 98 & 79.0 \\
\cline { 2 - 4 } & at night & 25 & 20.2 \\
\cline { 2 - 4 } & Day time & 1 & .8 \\
\cline { 2 - 4 } & Bathroom/toilet & 119 & 96.0 \\
\hline
\end{tabular}

Table 5: Housing/ building design of working places activity of samples

\begin{tabular}{|c|c|c|c|}
\hline & Variants & No. & Percentage \\
\hline \multirow[t]{3}{*}{ Levels of buildings } & One floor & 113 & 91.1 \\
\hline & Multiple floor & 0 & 0 \\
\hline & Underground & 11 & 8.9 \\
\hline \multirow[t]{3}{*}{ Construction materials } & Cement & 111 & 89.5 \\
\hline & Mud & 2 & 1.6 \\
\hline & Metal & 11 & 8.9 \\
\hline \multirow{2}{*}{$\begin{array}{l}\text { Household (habitat } \\
\text { status) }\end{array}$} & Room & 2 & 1.6 \\
\hline & Dormitories & 122 & 98.4 \\
\hline \multirow[t]{3}{*}{ Floor status } & Soiled & 115 & 92.7 \\
\hline & Tiled* & 2 & 1.6 \\
\hline & Cemented & 7 & 5.6 \\
\hline \multirow[t]{3}{*}{ Wall status } & Plastered & 2 & 1.6 \\
\hline & Rough & 47 & 37.9 \\
\hline & cracks crevices & 75 & 60.5 \\
\hline \multirow[t]{3}{*}{ Ceiling status } & Cement & 2 & 1.6 \\
\hline & Wood & 92 & 74.2 \\
\hline & Metal & 30 & 24.2 \\
\hline \multirow[t]{2}{*}{ Surrounding defence } & Low & 4 & 3.2 \\
\hline & Absent & 120 & 96.8 \\
\hline
\end{tabular}

Table 6: Time of insect bite and onset of lesions of samples

\begin{tabular}{|c|c|c|c|}
\hline & Months & No. & Percentage \\
\hline Time of insect bite & June & 76 & 61.3 \\
\cline { 2 - 4 } & July & 19 & 15.3 \\
\cline { 2 - 4 } & August & 3 & 2.4 \\
\cline { 2 - 4 } & not know & 26 & 21.0 \\
\hline \multirow{4}{*}{ Onset of lesion } & July & 2 & 1.6 \\
\cline { 2 - 4 } & August & 47 & 37.9 \\
\cline { 2 - 4 } & September & 64 & 51.6 \\
\cline { 2 - 4 } & Not know & 11 & $8.9 \%$ \\
\hline
\end{tabular}

Table 7: Initial complain, referral and diagnosis among samples

\begin{tabular}{|c|c|c|c|}
\hline & Variants & No. & Percentage \\
\hline \multirow{4}{*}{$\begin{array}{l}\text { Main presented } \\
\text { symptom }\end{array}$} & Asymptomatic (painless) & 39 & 31.5 \\
\hline & Painful & 15 & 12.1 \\
\hline & Itching & 1 & .8 \\
\hline & Disfiguring & 69 & 55.6 \\
\hline \multirow[t]{3}{*}{ Lesion discovering } & By himself & 93 & 75.0 \\
\hline & By colleagues & 23 & 18.5 \\
\hline & By work physician & 8 & 6.5 \\
\hline \multirow{2}{*}{$\begin{array}{l}\text { who referred } \\
(n=72)\end{array}$} & Responsible physician & 40 & 32.2 \\
\hline & Himself & 12 & 9.7 \\
\hline \multirow{3}{*}{$\begin{array}{l}\text { Presumptive diag- } \\
\text { nosis }\end{array}$} & allergy & 2 & 1.6 \\
\hline & Viral/bacterial infection & 7 & 5.6 \\
\hline & Clinically Suspected & 115 & 92.7 \\
\hline \multirow{3}{*}{$\begin{array}{l}\text { Method of definite } \\
\text { diagnosis }\end{array}$} & Clinical & 3 & 2.4 \\
\hline & Laboratory & 3 & 2.4 \\
\hline & Epidemiology to confirmed case & 118 & 95.2 \\
\hline
\end{tabular}

Missing $=52(41.9 \%)$ 
Table 8: Lesion characteristics among samples

\begin{tabular}{|l|c|c|c|}
\hline Lesion & Variants & No. & Percentage \\
\hline Type & Papule & 3 & 2.4 \\
\cline { 2 - 4 } & Nodule & 13 & 10.5 \\
\cline { 2 - 4 } & Ulcer & 106 & 85.5 \\
\cline { 2 - 4 } & Scar & 2 & 1.6 \\
\hline Size & $1 \times 1$ & 57 & 46.0 \\
\cline { 2 - 4 } & $2 \times 2$ & 64 & 51.6 \\
\cline { 2 - 4 } & More & 3 & 2.4 \\
\hline Site & Upper Limb & 46 & 37.1 \\
\cline { 2 - 4 } & Lower Limb & 69 & 55.6 \\
\cline { 2 - 4 } & Face & 8 & 6.5 \\
\cline { 2 - 4 } & Trunk & 1 & .8 \\
\hline Number & One & 24 & 19.4 \\
\cline { 2 - 4 } & Two & 62 & 50.0 \\
\cline { 2 - 4 } & $3-5$ & 26 & 21.0 \\
\cline { 2 - 4 } & $>5$ (Multiple) & 12 & 9.7 \\
\hline
\end{tabular}

Table 9: Treatment and outcome among samples

\begin{tabular}{|l|c|c|c|}
\hline Treatment & Treatment outcome & No. & Percentage \\
\hline Locally a-Fluconazole & Total received & 124 & 100 \\
\hline & Cured & 11 & 8.9 \\
\hline b-sodium stiboglyconate & Total received & 113 & 91.1 \\
\hline \multirow{4}{*}{ After referring } & Cured & 61 & 53.98 \\
\cline { 2 - 4 } & Surgical & 2 & 1.6 \\
\cline { 2 - 4 } & Cryotherapy & 16 & 12.9 \\
\cline { 2 - 4 } & Thermotherapy & 3 & 2.4 \\
\cline { 2 - 4 } & Laser & 19 & 15.3 \\
\cline { 2 - 4 } & No more treatment & 12 & 9.7 \\
\hline \multirow{5}{*}{ Outcome } & Cured & 108 & 87.1 \\
\cline { 2 - 4 } & Failed & 15 & 12.1 \\
\cline { 2 - 4 } & Adverse effect & 1 & .8 \\
\hline \multirow{3}{*}{ Complications up } & No & 96 & 77.4 \\
\cline { 2 - 4 } & Yes & 28 & 22.6 \\
\cline { 2 - 4 } & No complications & 75 & 60.5 \\
\cline { 2 - 4 } & Secondary infection & 15 & 12.1 \\
\cline { 2 - 4 } & Increase size & 15 & 12.1 \\
\cline { 2 - 4 } & Dark scar & 19 & 15.3 \\
\hline
\end{tabular}

Table 10: Lesion discovery and ZCL endemic knowledge among samples

\begin{tabular}{|l|c|c|c|c|c|}
\hline Discovery & \multicolumn{2}{|c|}{ No } & \multicolumn{2}{c|}{ Yes } & \\
\hline By himself & 86 & $76.1 \%$ & 7 & $63.6 \%$ & \multirow{2}{*}{0.249} \\
\hline By colleague & 21 & $18.6 \%$ & 2 & $18.2 \%$ \\
\hline By physician & 6 & $5.3 \%$ & 2 & $18.2 \%$ & \\
\hline Total & 113 & $100.0 \%$ & 11 & $100.0 \%$ & \\
\hline
\end{tabular}

Table 11: Lesion size and ZCL endemicity knowledge among samples.

\begin{tabular}{|c|c|c|c|c|c|c|}
\hline \multicolumn{2}{|c|}{ Lesion } & \multicolumn{2}{|c|}{ No } & \multicolumn{2}{|c|}{ Yes } & \multirow{2}{*}{ P value } \\
\cline { 2 - 7 } & & No. & $\%$ & No. & $\%$ & \\
\hline \multirow{3}{*}{ size } & $1 \times 1$ & 50 & $44.2 \%$ & 7 & $63.6 \%$ & \\
\cline { 2 - 6 } & $2 \times 2$ & 61 & $54.0 \%$ & 3 & $27.3 \%$ & \multirow{3}{*}{0.109} \\
\cline { 2 - 6 } & more & 2 & $1.8 \%$ & 1 & $9.1 \%$ & \\
\hline \multicolumn{2}{|c|}{ Total } & 113 & $100.0 \%$ & 11 & $100.0 \%$ & \\
\hline
\end{tabular}

Table 12: Lesion site and ZCL endemicity knowledge among samples.

\begin{tabular}{|l|c|c|c|c|c|}
\hline \multirow{2}{*}{ Lesion site } & \multicolumn{2}{|c|}{ No } & \multicolumn{2}{c|}{ Yes } & \multirow{2}{*}{ P value } \\
\cline { 2 - 5 } & No. & $\%$ & No. & $\%$ & \\
\hline Upper limb & 45 & $39.8 \%$ & 1 & $9.1 \%$ & \\
\hline Lower limb & 61 & $54.0 \%$ & 8 & $72.7 \%$ & \multirow{2}{*}{0.004} \\
\hline Face & 7 & $6.2 \%$ & 1 & $9.1 \%$ & \\
\hline Trunk & 0 & $0.0 \%$ & 1 & $9.1 \%$ & \\
\hline Total & 113 & $100.0 \%$ & 11 & $100.0 \%$ & \\
\hline
\end{tabular}


Table 13: Number of lesions and ZCL endemicity knowledge among samples.

\begin{tabular}{|c|c|c|c|c|c|}
\hline \multirow{2}{*}{$\begin{array}{l}\text { Lesion } \\
\text { number }\end{array}$} & \multicolumn{2}{|c|}{ No } & \multicolumn{2}{|c|}{ Yes } & \multirow{2}{*}{$P$ value } \\
\hline & No. & $\%$ & No. & $\%$ & \\
\hline one & 23 & $20.4 \%$ & 1 & $9.1 \%$ & \multirow{4}{*}{0.475} \\
\hline Two & 55 & $48.7 \%$ & 7 & $63.6 \%$ & \\
\hline $3-5$ & 23 & $20.4 \%$ & 3 & $27.3 \%$ & \\
\hline$>5$ (multible) & 12 & $10.6 \%$ & 0 & $0.0 \%$ & \\
\hline Total & 113 & $100.0 \%$ & 11 & $100.0 \%$ & \\
\hline
\end{tabular}

Table 14: Association between lesions characteristics and age groups

\begin{tabular}{|c|c|c|c|c|c|c|}
\hline Lesion & Picture & \multicolumn{2}{|c|}{$20-30$ year } & \multicolumn{2}{|c|}{$>30$} & P value \\
\hline Type & Papule & 3 & $2.6 \%$ & 0 & $0.0 \%$ & \\
\cline { 2 - 6 } & Nodule & 10 & $8.5 \%$ & 3 & $42.9 \%$ & \multirow{4}{*}{0.038} \\
\cline { 2 - 6 } & Ulcer & 102 & $87.2 \%$ & 4 & $57.1 \%$ & \\
\cline { 2 - 6 } & Scar & 2 & $1.7 \%$ & 0 & $0.0 \%$ & \\
\hline Size & $1 \times 1$ & 51 & $43.6 \%$ & 6 & $85.7 \%$ & \multirow{4}{*}{0.094} \\
\cline { 2 - 6 } & $2 \times 2$ & 63 & $53.8 \%$ & 1 & $14.3 \%$ & \\
\cline { 2 - 6 } & more & 3 & $2.6 \%$ & 0 & $0.0 \%$ & \\
\hline Site & upper limb & 44 & $37.6 \%$ & 2 & $28.6 \%$ & \\
\cline { 2 - 6 } & lowe limb & 64 & $54.7 \%$ & 5 & $71.4 \%$ & \multirow{4}{*}{0797} \\
\cline { 2 - 6 } & face & 8 & $6.8 \%$ & 0 & $0.0 \%$ & \\
\cline { 2 - 6 } & trunk & 1 & $0.9 \%$ & 0 & $0.0 \%$ & \\
\hline No. & one & 23 & $19.7 \%$ & 1 & $14.3 \%$ & \\
\cline { 2 - 6 } & two & 56 & $47.9 \%$ & 6 & $85.7 \%$ & \multirow{3}{*}{0.234} \\
\cline { 2 - 6 } & $3-5$ & 26 & $22.2 \%$ & 0 & $0.0 \%$ & \\
\cline { 2 - 6 } & $>5($ multible) & 12 & $10.3 \%$ & 0 & $0.0 \%$ & \\
\hline
\end{tabular}

Table 15: Association between lesions characteristics and patient's rank

\begin{tabular}{|c|c|c|c|c|c|c|c|c|}
\hline \multicolumn{2}{|r|}{ Lesion } & \multicolumn{2}{|c|}{ Officer } & \multicolumn{2}{|c|}{ Warrant officer } & \multicolumn{2}{|c|}{ Manual workers } & $\mathrm{P}$ value \\
\hline \multirow[t]{4}{*}{ Type } & \begin{tabular}{|l|} 
Papule \\
\end{tabular} & 0 & $0.0 \%$ & 0 & $0.0 \%$ & 3 & $2.7 \%$ & \multirow{4}{*}{0.433} \\
\hline & Nodule & 0 & $0.0 \%$ & 3 & $33.3 \%$ & 10 & $8.8 \%$ & \\
\hline & Ulcer & 2 & $100.0 \%$ & 6 & $66.7 \%$ & 98 & $86.7 \%$ & \\
\hline & Scar & 0 & $0.0 \%$ & 0 & $0.0 \%$ & 2 & $1.8 \%$ & \\
\hline \multirow[t]{3}{*}{ Size } & $1 \times 1$ & 0 & $0.0 \%$ & 7 & $77.8 \%$ & 50 & $44.2 \%$ & \multirow{3}{*}{0.220} \\
\hline & $2 \times 2$ & 2 & $100.0 \%$ & 2 & $22.2 \%$ & 60 & $53.1 \%$ & \\
\hline & More & 0 & $0.0 \%$ & 0 & $0.0 \%$ & 3 & $2.7 \%$ & \\
\hline \multirow[t]{4}{*}{ Site } & Upper limb & 1 & $50.0 \%$ & 2 & $22.2 \%$ & 43 & $38.1 \%$ & \multirow{4}{*}{0.881} \\
\hline & Lower limb & 1 & $50.0 \%$ & 7 & $77.8 \%$ & 61 & $54.0 \%$ & \\
\hline & Face & 0 & $0.0 \%$ & 0 & $0.0 \%$ & 8 & $7.1 \%$ & \\
\hline & \begin{tabular}{|l|} 
Trunk \\
\end{tabular} & 0 & $0.0 \%$ & 0 & $0.0 \%$ & 1 & $0.9 \%$ & \\
\hline \multirow[t]{4}{*}{ No. } & One & 0 & $0.0 \%$ & 1 & $11.1 \%$ & 23 & $20.4 \%$ & \multirow{4}{*}{0.411} \\
\hline & Two & 2 & $100.0 \%$ & 7 & $77.8 \%$ & 53 & $46.9 \%$ & \\
\hline & $3-5$ & 0 & $0.0 \%$ & 0 & $0.0 \%$ & 26 & $23.0 \%$ & \\
\hline & $>5$ (multible) & 0 & $0.0 \%$ & 1 & $11.1 \%$ & 11 & $9.7 \%$ & \\
\hline
\end{tabular}

Table 16: Association between lesions characteristics and previous occupation

\begin{tabular}{|c|c|c|c|c|c|c|c|c|c|c|}
\hline \multicolumn{2}{|r|}{ Lesion } & \multicolumn{2}{|c|}{ Student } & \multicolumn{2}{|c|}{ Farmer } & \multicolumn{2}{|c|}{ Laborer } & \multicolumn{2}{|c|}{ Administrative worker } & \multirow[t]{2}{*}{$\mathrm{P}$ value } \\
\hline \multirow{4}{*}{ Type } & Papule & 2 & $4.3 \%$ & 0 & $0.0 \%$ & 1 & $6.7 \%$ & 0 & $0.0 \%$ & \\
\hline & Nodule & 7 & $14.9 \%$ & 3 & $6.3 \%$ & 1 & $6.7 \%$ & 2 & $14.3 \%$ & \multirow{3}{*}{0.421} \\
\hline & Ulcer & 38 & $80.9 \%$ & 44 & $91.7 \%$ & 12 & $80.0 \%$ & 12 & $85.7 \%$ & \\
\hline & \begin{tabular}{|l|} 
Scar \\
\end{tabular} & 0 & $0.0 \%$ & 1 & $2.1 \%$ & 1 & $6.7 \%$ & 0 & $0.0 \%$ & \\
\hline \multirow{3}{*}{ Size } & $1 \times 1$ & 18 & $38.3 \%$ & 20 & $41.7 \%$ & 9 & $60.0 \%$ & 10 & $71.4 \%$ & \multirow{3}{*}{0.302} \\
\hline & $2 \times 2$ & 28 & $59.6 \%$ & 26 & $54.2 \%$ & 6 & $40.0 \%$ & 4 & $28.6 \%$ & \\
\hline & More & 1 & $2.1 \%$ & 2 & $4.2 \%$ & 0 & $0.0 \%$ & 0 & $0.0 \%$ & \\
\hline \multirow{4}{*}{ Site } & Upper limb & 19 & $40.4 \%$ & 19 & $39.6 \%$ & 3 & $20.0 \%$ & 5 & $35.7 \%$ & \multirow{4}{*}{0.044} \\
\hline & Lower limb & 28 & $59.6 \%$ & 24 & $50.0 \%$ & 8 & $53.3 \%$ & 9 & $64.3 \%$ & \\
\hline & Face & 0 & $0.0 \%$ & 4 & $8.3 \%$ & 4 & $26.7 \%$ & 0 & $0.0 \%$ & \\
\hline & \begin{tabular}{|l|} 
Trunk \\
\end{tabular} & 0 & $0.0 \%$ & 1 & $2.1 \%$ & 0 & $0.0 \%$ & 0 & $0.0 \%$ & \\
\hline \multirow{4}{*}{ No. } & One & 12 & $25.5 \%$ & 7 & $14.6 \%$ & 4 & $26.7 \%$ & 1 & $7.1 \%$ & \multirow{4}{*}{0.353} \\
\hline & Two & 24 & $51.1 \%$ & 21 & $43.8 \%$ & 7 & $46.7 \%$ & 10 & $71.4 \%$ & \\
\hline & $3-5$ & 9 & $19.1 \%$ & 13 & $27.1 \%$ & 3 & $20.0 \%$ & 1 & $7.1 \%$ & \\
\hline & $>5$ (multiple) & 2 & $4.3 \%$ & 7 & $14.6 \%$ & 1 & $6.7 \%$ & 2 & $14.3 \%$ & \\
\hline
\end{tabular}


Table 17: Association between lesions characteristics and patient's education level

\begin{tabular}{|c|c|c|c|c|c|c|c|c|}
\hline & \multirow{2}{*}{\begin{tabular}{l|l} 
Lesion \\
Papule
\end{tabular}} & \multicolumn{2}{|c|}{ Read \& write } & \multicolumn{2}{|c|}{ Middle education } & \multicolumn{2}{|c|}{ High education } & \multirow[t]{2}{*}{$\mathrm{P}$ value } \\
\hline \multirow{4}{*}{ Type } & & 0 & $0.0 \%$ & 3 & $2.8 \%$ & 0 & $0.0 \%$ & \\
\hline & Nodule & 0 & $0.0 \%$ & 11 & $10.4 \%$ & 2 & $15.4 \%$ & \multirow{3}{*}{0.937} \\
\hline & Ulcer & 5 & $100.0 \%$ & 90 & $84.9 \%$ & 11 & $84.6 \%$ & \\
\hline & Scar & 0 & $0.0 \%$ & 2 & $1.9 \%$ & 0 & $0.0 \%$ & \\
\hline \multirow{3}{*}{ Size } & $1 \times 1$ & 3 & $60.0 \%$ & 43 & $40.6 \%$ & 11 & $84.6 \%$ & \multirow{3}{*}{0.048} \\
\hline & $2 \times 2$ & 2 & $40.0 \%$ & 60 & $56.6 \%$ & 2 & $15.4 \%$ & \\
\hline & More & 0 & $0.0 \%$ & 3 & $2.8 \%$ & 0 & $0.0 \%$ & \\
\hline \multirow{4}{*}{ Site } & Upper limb & 1 & $20.0 \%$ & 38 & $35.8 \%$ & 7 & $53.8 \%$ & \multirow{4}{*}{0.718} \\
\hline & Lower limb & 4 & $80.0 \%$ & 59 & $55.7 \%$ & 6 & $46.2 \%$ & \\
\hline & Face & 0 & $0.0 \%$ & 8 & $7.5 \%$ & 0 & $0.0 \%$ & \\
\hline & Trunk & 0 & $0.0 \%$ & 1 & $0.9 \%$ & 0 & $0.0 \%$ & \\
\hline \multirow{4}{*}{ No. } & one & 1 & $20.0 \%$ & 21 & $19.8 \%$ & 2 & $15.4 \%$ & \multirow{4}{*}{0.706} \\
\hline & two & 3 & $60.0 \%$ & 52 & $49.1 \%$ & 7 & $53.8 \%$ & \\
\hline & $3-5$ & 0 & $0.0 \%$ & 22 & $20.8 \%$ & 4 & $30.8 \%$ & \\
\hline & $>5$ (multiple) & 1 & $20.0 \%$ & 11 & $10.4 \%$ & 0 & $0.0 \%$ & \\
\hline
\end{tabular}

Table 18: Association between lesions characteristics and sleeping pattern

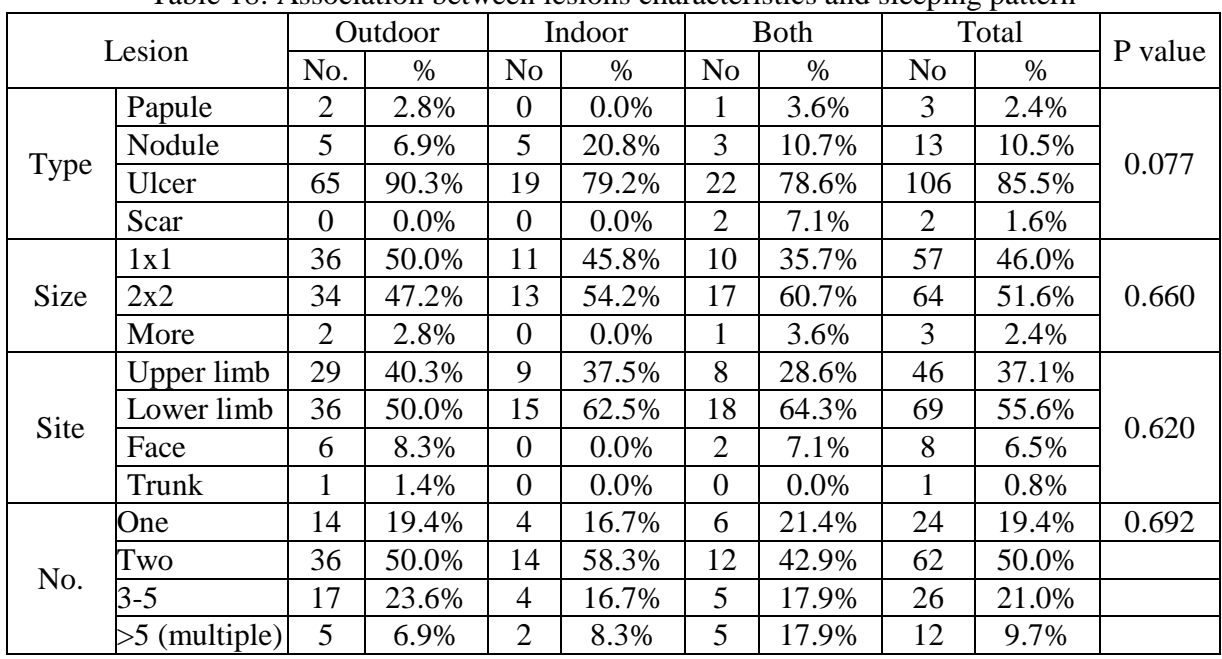

Table 19: Outcome and sequellae treatment with sleeping pattern

\begin{tabular}{|l|c|c|c|c|c|c|c|c|c|}
\hline \multirow{2}{*}{$\begin{array}{l}\text { Treatment } \\
\text { Outcome }\end{array}$} & \multicolumn{2}{|c|}{ Outdoor } & \multicolumn{2}{|c|}{ Indoor } & \multicolumn{2}{|c|}{ Both } & \multicolumn{2}{|c|}{ Total } & \multirow{2}{*}{ P value } \\
\cline { 2 - 10 } & No. & $\%$ & No. & $\%$ & No. & $\%$ & No. & $\%$ & \\
\hline Cured & 63 & 87.5 & 22 & 91.7 & 23 & 82.1 & 108 & 87.1 & \multirow{2}{*}{0.410} \\
\hline Failed & 9 & 12.5 & 2 & 8.3 & 4 & 14.3 & 15 & 12.1 & \\
\hline Adverse effect & 0 & 0.0 & 0 & 0.0 & 1 & 3.6 & 1 & 0.8 & \\
\hline No side effect & 42 & 58.3 & 16 & 66.7 & 17 & 60.7 & 75 & 60.5 & \multirow{2}{*}{0.985} \\
\hline 2ry infection & 10 & 13.9 & 2 & 8.3 & 3 & 10.7 & 15 & 12.1 & \\
\hline Increase size & 9 & 12.5 & 3 & 12.5 & 3 & 10.7 & 15 & 12.1 \\
\hline Scar & 11 & 15.3 & 3 & 12.5 & 5 & 17.9 & 19 & 15.3 & \\
\hline
\end{tabular}

Table 20: Association between work characteristics and outcome

\begin{tabular}{|c|c|c|c|c|c|c|c|c|c|c|}
\hline & \multicolumn{2}{|c|}{ Cured } & \multicolumn{2}{|c|}{ Failed } & \multicolumn{2}{|c|}{ Adverse effect } & \multicolumn{2}{|c|}{ Total } & \multirow{2}{*}{$P$ value } \\
\hline & & No & $\%$ & No & $\%$ & No & $\%$ & No & $\%$ & \\
\hline \multirow{3}{*}{$\begin{array}{l}\text { Original/ } \\
\text { residence }\end{array}$} & Upper Egypt & 68 & 63.0 & 7 & 46.7 & 1 & 100.0 & 76 & 61.3 & \multirow{3}{*}{0.384} \\
\hline & Lower Egypt & 26 & 24.1 & 7 & 46.7 & 0 & 0.0 & 33 & 26.6 & \\
\hline & Suez canal zone & 14 & 13.0 & 1 & 6.7 & 0 & 0.0 & 15 & 12.1 & \\
\hline \multirow{3}{*}{$\begin{array}{l}\text { Length of } \\
\text { stay in } \\
\text { months }\end{array}$} & $<6 \mathrm{mo}$ & 5 & 4.6 & 0 & 0.0 & 0 & 0.0 & 5 & 4.0 & \multirow{3}{*}{0.867} \\
\hline & $6-12 \mathrm{mo}$ & 106 & 93 & 15 & 100.0 & 1 & 100.0 & 116 & 93.5 & \\
\hline & $>12 \mathrm{mo}$ & 3 & 2.8 & 0 & 0.0 & 0 & 0.0 & 3 & 2.4 & \\
\hline \multirow{3}{*}{$\begin{array}{c}\text { Nature of } \\
\text { work }\end{array}$} & Stable & 94 & 87.0 & 13 & 86.7 & 1 & 100.0 & 108 & 87.1 & \multirow{3}{*}{0.996} \\
\hline & Movable & 8 & 7.4 & 1 & 6.7 & 0 & 0.0 & 9 & 7.3 & \\
\hline & Variable/mix & 6 & 5.6 & 1 & 6.7 & 0 & 0.0 & 7 & 5.6 & \\
\hline \multirow{3}{*}{ Current task } & Manual services & 98 & 90.7 & 14 & 93.3 & 1 & 100.0 & 113 & 91.1 & \multirow{3}{*}{0.983} \\
\hline & \begin{tabular}{|l|} 
Logistic services \\
\end{tabular} & 8 & 7.4 & 1 & 6.7 & 0 & 0.0 & 9 & 7.3 & \\
\hline & Deputy & 2 & 1.9 & 0 & 0.0 & 0 & 0.0 & 2 & 1.6 & \\
\hline
\end{tabular}


Table 21; Prevention ZCL knowledge and practices and treatment outcome

\begin{tabular}{|c|c|c|c|c|c|c|c|c|c|c|}
\hline \multirow{2}{*}{\multicolumn{2}{|c|}{$\begin{array}{l}\text { Prevention ZCL } \\
\text { knowledge }\end{array}$}} & \multicolumn{2}{|c|}{ Cured } & \multicolumn{2}{|c|}{ Failed } & \multicolumn{2}{|c|}{ Adverse effect } & \multicolumn{2}{|c|}{ Total } & \multirow{2}{*}{$P$ value } \\
\hline & & No & $\%$ & No & $\%$ & No & $\%$ & No & $\%$ & \\
\hline & No & 99 & 91.7 & 13 & 86.7 & 1 & 100.0 & 113 & 91.1 & 0.651 \\
\hline & Yes & 4 & 3.7 & 0 & 0.0 & 0 & 0.0 & 4 & 3.2 & \\
\hline & Didn't know & 5 & 4.6 & 2 & 13.3 & 0 & 0.0 & 7 & 5.6 & \\
\hline \multirow{3}{*}{$\begin{array}{l}\text { Sleeping } \\
\text { pattern }\end{array}$} & Outdoor & 63 & 58.3 & 9 & 60.0 & 0 & 0.0 & 72 & 58.1 & 0.410 \\
\hline & Indoor & 22 & 20.4 & 2 & 13.3 & 0 & 0.0 & 24 & 19.4 & \\
\hline & Both & 23 & 21.3 & 4 & 26.7 & 1 & 100.0 & 28 & 22.6 & \\
\hline \multirow{3}{*}{$\begin{array}{l}\text { Sleeping } \\
\text { time }\end{array}$} & Dusk & 57 & 52.8 & 9 & 60.0 & 1 & 100.0 & 67 & 54.0 & 0.876 \\
\hline & Mid-night & 22 & 20.4 & 3 & 20.0 & 0 & 0.0 & 25 & 20.2 & \\
\hline & Dawn & 29 & 26.9 & 3 & 20.0 & 0 & 0.0 & 32 & 25.8 & \\
\hline \multirow{2}{*}{ Repellant } & No & 82 & 75.9 & 12 & 80.0 & 0 & 0.0 & 94 & 75.8 & 0.194 \\
\hline & Not Know & 26 & 24.1 & 3 & 20.0 & 1 & 100.0 & 30 & 24.2 & \\
\hline \multirow{2}{*}{$\begin{array}{l}\text { Bed nets } \\
\text { or cover }\end{array}$} & No & 63 & 58.3 & 7 & 46.7 & 0 & 0.0 & 70 & 56.5 & 0.361 \\
\hline & Yes & 45 & 41.7 & 8 & 53.3 & 1 & 100.0 & 54 & 43.5 & \\
\hline
\end{tabular}

Table 22: Prevention ZCL knowledge and practices and site of lesion

\begin{tabular}{|c|c|c|c|c|c|c|c|c|c|c|c|}
\hline \multirow{2}{*}{$\begin{array}{l}\text { Prevention ZCL } \\
\text { knowledge }\end{array}$} & \multicolumn{2}{|c|}{ Upper limb } & \multicolumn{2}{|c|}{ Lower limb } & \multicolumn{2}{|c|}{ Face } & \multicolumn{2}{|c|}{ Trunk } & \multicolumn{2}{|c|}{ Total } & \multirow{2}{*}{$\begin{array}{c}\mathrm{P} \\
\text { value }\end{array}$} \\
\hline & No & $\%$ & No & $\%$ & No & $\%$ & No & $\%$ & No & $\%$ & \\
\hline No & 45 & 97.8 & 61 & 88.4 & 7 & 87.5 & 0 & 0.0 & 113 & 91.1 & \multirow{3}{*}{0.002} \\
\hline Yes & 1 & 2.2 & 3 & 4.3 & 0 & 0.0 & 0 & 0.0 & 4 & 3.2 & \\
\hline Didn't know & 0 & 0.0 & 5 & 7.2 & 1 & 12.5 & 1 & 100.0 & 7 & 5.6 & \\
\hline
\end{tabular}

Table 23: Sleep pattern and time with site of lesion

\begin{tabular}{|c|c|c|c|c|c|c|c|c|c|c|c|}
\hline & \multicolumn{2}{|c|}{ Upper limb } & \multicolumn{2}{|c|}{ Lowe limb } & \multicolumn{2}{|c|}{ Face } & \multicolumn{2}{|c|}{ Trunk } & \multicolumn{2}{|c|}{ Total } & \multirow{2}{*}{$\begin{array}{c}\mathrm{P} \\
\text { value }\end{array}$} \\
\hline & No & $\%$ & No & $\%$ & No & $\%$ & No & $\%$ & No & $\%$ & \\
\hline Sleeping Outdoor & 29 & 63.0 & 36 & 52.2 & 6 & 75.0 & 1 & 100.0 & 72 & 58.1 & \multirow{3}{*}{0.620} \\
\hline Sleeping Indoor & 9 & 19.6 & 15 & 21.7 & 0 & 0.0 & 0 & 0.0 & 24 & 19.4 & \\
\hline Both & 8 & 17.4 & 18 & 26.1 & 2 & 25.0 & 0 & 0.0 & 28 & 22.6 & \\
\hline Time Dusk & 31 & 67.4 & 33 & 47.8 & 2 & 25.0 & 1 & 100.0 & 67 & 54.0 & \multirow{3}{*}{0.182} \\
\hline Time Mid-ni & 8 & 17.4 & 15 & 21.7 & 2 & 25.0 & 0 & 0.0 & 25 & 20.2 & \\
\hline Time Dawn & 7 & 15.2 & 21 & 30.4 & 4 & 50.0 & 0 & 0.0 & 32 & 25.8 & \\
\hline
\end{tabular}

Table 24: Using bed-nets or cover and lesion's size

\begin{tabular}{|c|c|c|c|c|c|c|c|c|}
\hline \multirow{2}{*}{\multicolumn{2}{|c|}{ Lesion }} & \multicolumn{2}{|c|}{ No } & \multicolumn{2}{|c|}{ Yes } & \multicolumn{2}{|c|}{ Total } & \multirow{2}{*}{$\begin{array}{c}\mathrm{P} \\
\text { value }\end{array}$} \\
\hline & & No & $\%$ & No & $\%$ & No & $\%$ & \\
\hline \multirow{3}{*}{ Size } & $1 \times 1$ & 27 & 38.6 & 30 & 55.6 & 57 & 46.0 & \multirow{3}{*}{0.093} \\
\hline & $2 \times 2$ & 42 & 60.0 & 22 & 40.7 & 64 & 51.6 & \\
\hline & More & 1 & 1.4 & 2 & 3.7 & 3 & 2.4 & \\
\hline
\end{tabular}

Table 25: Using bed nets or cover and lesion's site

\begin{tabular}{|c|c|c|c|c|c|c|c|c|}
\hline \multirow{2}{*}{\multicolumn{2}{|c|}{ Lesion }} & \multicolumn{2}{|c|}{ No } & \multicolumn{2}{|c|}{ Yes } & \multicolumn{2}{|c|}{ Total } & \multirow{2}{*}{$\begin{array}{c}\mathrm{P} \\
\text { value }\end{array}$} \\
\hline & & No & $\%$ & No & $\%$ & No & $\%$ & \\
\hline \multirow{4}{*}{ Site } & Upper limb & 25 & 35.7 & 21 & 38.9 & 46 & 37.1 & \multirow{4}{*}{0.668} \\
\hline & Lower limb & 40 & 57.1 & 29 & 53.7 & 69 & 55.6 & \\
\hline & Face & 5 & 7.1 & 3 & 5.6 & 8 & 6.5 & \\
\hline & Trunk & 0 & 0.0 & 1 & 19 & 1 & & \\
\hline
\end{tabular}

Table 26: Using bed nets or cover and number of lesion

\begin{tabular}{|c|c|c|c|c|c|c|c|}
\hline \multirow{2}{*}{ Lesion } & \multicolumn{2}{|c|}{ No } & \multicolumn{2}{|c|}{ Yes } & \multicolumn{2}{|c|}{ Total } & \multirow{2}{*}{$\begin{array}{c}\mathrm{P} \\
\text { value }\end{array}$} \\
\hline & No. & $\%$ & No. & $\%$ & No. & $\%$ & \\
\hline One & 14 & 20.0 & 10 & 18.5 & 24 & 19.4 & \multirow{4}{*}{0.394} \\
\hline Two & 36 & 51.4 & 26 & 48.1 & 62 & 50.0 & \\
\hline $3-5$ & 16 & 22.9 & 10 & 18.5 & 26 & 21.0 & \\
\hline$>5$ (multiple) & 4 & 5.7 & 8 & 14.8 & 12 & 9.7 & \\
\hline
\end{tabular}

Table 27: Surrounding area and vector breeding place

\begin{tabular}{|c|c|c|c|c|c|c|c|}
\hline \multirow{2}{*}{ Breeding site } & \multicolumn{2}{|c|}{ Desert } & \multicolumn{2}{|c|}{ Residential } & \multicolumn{2}{|c|}{ Total } & \multirow{2}{*}{$\begin{array}{c}\mathrm{P} \\
\text { value }\end{array}$} \\
\hline & No. & $\%$ & No. & $\%$ & No. & $\%$ & \\
\hline Bathroom/toilet & 115 & 96.6 & 4 & 80. & 119 & 96.0 & \multirow{3}{*}{0.000} \\
\hline Waste & 4 & 3.4 & 0 & 0.0 & 4 & 3.2 & \\
\hline Vegetation & 0 & 0.0 & 1 & 20 & 1 & 0.8 & \\
\hline
\end{tabular}


Table 28: Predictors of treatment failure among samples.

\begin{tabular}{|l|c|c|c|}
\hline & B & Sig. & Exp. (B) \\
\hline Constant & -18.090 & .999 & .000 \\
\hline Occupation (administrative worker/ students) & 3.388 & .002 & 29.595 \\
\hline Education (middle education/ read and write) & -2.923 & .017 & .054 \\
\hline Building design (underground/one floor & 1.331 & .050 & 3.784 \\
\hline Lesion discovering (by colleague or himself) & 1.215 & .044 & 3.369 \\
\hline Complications (increase size/ none) & 2.319 & .005 & 10.160 \\
\hline
\end{tabular}

\section{Discussion}

Zoonotic cutaneous leishmaniasis is a neglected clinical form that is highly prevalent in the northern Sinai in Egypt. This is true since the last studied on the rodents (Morsy et al, 1987) on man (Morsy et al, 1992) and on the sand fly vector (Hamadto et al, 2007). It represented one of the important public health problems in many parts of the world, especially the Mediterranean and Middle East Countries (Shehata et al, 2009). Studies of (CL) in Egypt were relevant and important for the following reasons: 1- Egypt presents district environmental, geographic and demographic contexts as compared to other regional countries that are more frequently studied. 2- Egypt represents the regional crossroads of many countries and a potential source of infection from and to neighboring countries. 3- Egypt has experienced extreme weather events in recent years. 4- Wide deployment of manual workers coming from the Nile Vallry mainly in North Sinai, a known endemic district.

Risk assessment was defined as a systematic process of evaluating and measuring the potential risks that may be involved in acquiring cutaneous leishmaniasis, so, the present study planned to meet the following inquiries: 1- Is there potential risk or not? 2What are the major risk factors? 3- Estimating the existing risks as in frequent, frequent or more frequent and 4- Who are at risk and rating them as low, moderate or high risk groups.

The present study results showed that the great majority of cases (94\%) were aged between (20-30) years old, all were males, the most affected cases originally residing in rural areas in Upper Egypt (61\%), and then Lower Egypt. These finding were essential without significant age, sex or residence bi- as, Also, it was found that the majority of cases $(85 \%)$ were in middle education class, previously working as farmers and students with about $38 \%$ for each, these results agreed with Ranjan et al. (2005) in India and Abdellatif et al. (2012) and Ahmed and Abou Faddan (2013) in Libya where CL was significantly associated with illiteracy and farm working as occupational disease.

The current study showed that most of cases $(93.5 \%)$ stayed more than 6 month, they are working in stable tasks $(91.1 \%)$ that enhanced the longstanding exposure of susceptible persons to sand fly vectors, which agreed with Peterson (2008) who stated that all components of disease transmission systems are required to permit long term circulation in a region.

As regards the individual practices and knowledge, the majority of cases $(91 \%)$ were not informed about endemicity and more than half $(58 \%)$ slept outdoor at the dusk time coinciding with preferred time of sand-fly activities. This data agreed with Klemper et al. (2007) who reported that the female sand-fly needs blood in order to obtain the protein necessary to develop its eggs; they bite especially at night and dusk. Ahmed and Abo Faddan (2013) in Libya reported that the involved people were engaged in the occupational activities working outdoors to night, the present study showed majority of the patients neither used insect repellent nor bed nets.

The present sites of lesions were significantly influenced by the lack of endemicity knowledge of the strict protective measures such as (bednets, insect repellent regularly and avoiding sleep outdoor especially at dusk time), which agreed with AbduIsadah (2011) in Iraq. The use of the textile fabrics whether insecticide-treated or not, as bed- 
nets reduced indoor ZCL transmission in the endemic areas (Yaghoobi-Ershadi et al, 2006; Mossa-Kazermi et al, 2007). However, bed nets as protective agent dependent on whether they were insecticide impregnated, their pores per inch, size, or wear and tear (Das et al, 2007).

The present study found that sandy soil, mountain and desert were surrounding the work place $(100 \%, 97 \%$ \& 96\%) respectively associated with the desert vegetation as Chenopodium species. This genus (Chenopodium), Family Chenopodiaceae) is native to Hawaii, Southern America, Asia-Tropical, Asia-Temperate, Africa, Northern America, Australasia and Europe, containing at least 150 annual or perennial species distributed worldwide (Fuentes-Bazan et al, 2012). The Chenopodium ambrosioides (Morsy et al, 1998) and Ch. quinoa Willd are the species common in the Egyptian desert (Eisa et al, 2014). The Psammomys obesus Cretzschmar 1828 proved the main animal reservoir for ZCL in the old world (Morsy et al, 1996). Its habitats in Egypt are saline soils and salt marches with stands of succulent halophytic vegetation chiefly of family Chenopodeiaceae Osbern and Helmy, 1980). Apart from $P$. obeswus, Gerbillus pyramidum proved to be other hosts of ZCL in the Sinai Peninsula (Morsy et al, 1987b) as well as Meriones crassus (Morsy et al, 1991) and Meriones sacramenti Thomas 1992 (Morsy et al, 1993). Moreover, natural infections of $L$. major in domestic dogs from Alexandria (Morsy et al, 1987c), and the red fox Vulpes $O$. aegyptica was an animal reservoir for ZCL (Morsy et al, 2002).

In the present study, $79 \%$ of cases did not recognize sand flies in or outdoors but reported painless bites. This agreed with Voisin et al. (2011). The main breeding places were within and around the area of bathroom (96\%). This agreed with Lewis (1971) who found that the scattering of sand flies at a certain area, was dependent on availability of water and dampness.

In the present study, there was significant- ly influenced by housing condition. This agreed with Ahmed and Abou Faddan (2013) who found that CL was significantly associated with first floors and few rooms per house and their small size enables them to live in various different microhabitats such as cracks, crevices and holes (Feliciangeli et al, 2006). In the current study, sandfly bites were in June but lesions appeared at the end of August (37.9\%) and beginning of September (51.6\%). Bruce and John (2004) attributed seasonal abundance of sand files, to significant seasonal fluctuation in climate, where sand fly populations were highest toward the end of the rainy season and lowest toward the end of the dry seasons. Fahmy et al. (2010) in Egypt found that L. major infections circulated in the country through different seasons of the year with i peaks in November, March, August and few sporadic cases were reported during the rest of the year

The present study showed that ulcer was the commonest clinical presentation (85\%), measuring approximately $(2 \times 2 \mathrm{~cm})$ in size and more common in exposed parts (upper, lower limb) with marked increasing in lower limb. The small sized lesion $(1 \times 1 \mathrm{~cm})$ was insignificantly associated with endemicity knowledge $(63 \%)$, while larger size lesions correlated to lack of endemicity knowledge (54\%) without statistical significant difference.

In this study, fluconazole treated11/124 (8.9\%), while Nastiboglucanate (Pentostam) was more effective therapy $61 / 113(53.9 \%)$ however, it has poor compliance because of its parenteral administration compared to fluconazole which is taken in oral way, on contrary to report of WHO (2010) as regards lines of treatment, fluconazole is considered the least effective therapy for CL. AbdelMotagaly and Morsy (2016) in Egypt reported that a total of 126 patients with zoonotic cutaneous were referred to the Military Fever Hospital (74 in 2013; 47 in 2014 and 5 in 2015). The majority of these patients had multiplelesions $90 \%$, on the lower limbs and 
upper limbs but, rare on face, mainly on the ears. The lesions with the secondary bacterial infections were treated by the antibiotics, Fluconazole $^{\circledR} 5 \mathrm{mg} / \mathrm{kg}$ orally once per daily for four weeks. They added that some complicated lesions required another course (73\%) and followed up to the liver function tests. Complicated cases of more than $2 / 3$ lesions with $40 \mathrm{~mm}$ diameter, cosmetic problem, LN spread. Some cases $(70 \%)$ with the complicated lesions after the second course of Fluconazole ${ }^{\circledR}$ were offered sodium stibogluconate (Pentostam ${ }^{\circledR}$ ) and/or the cryosurgery (Morsy et al, 1989).

Generally speaking, the ZCL is a public health threatened to Egypt, particularly Sinai Peninsula. Two ZCL foci were identified in North Sinai (Faris et al, 1988) and presence of Egyptian ZCL patient manifested as diffuse CL (Morsy et al, 1997) at least nine species sandflies were encountered in Sinai (El Sawaf et al, 1987) and the Nile Delta (Morsy et al, 1990) as well as allover many Egyptian areas (Saleh et al, 2015). Among these sand-flies were P. papatasii (Scopoli), the vector of ZCL (Wahba et al, 1990) and $P$. sergenti, the vector of ACL (Hanafi et al, 2001). As to the rodents the reservoir hosts of ZCL, Order Rodentia included a total of 72 Genera according to the survey carried allover Egypt (Osborn and Helmy, 1980). Morsy et al. (1993) gave an illustrated key for the rodents of medical importance in Sinai Peninsula. Of interest, Psammomys obesus Cretzchmar, 1828 the main reservoir host for ZCL in the Eastern Mediterranean Countries was detected a in all the Egyptian deserts (Morsy et al, 1996) and the wild rodents Gerbillus pyramidiun (Morsy et al, 1987b), Meriones crassus (Morsy et al, 1991) and Meriones sacramenti Thomas, 1992 (Morsy et al, 1993) proved to be reservoir hosts as well allover Sinai, Suez Canal Zone and the North Costal Region. Also, endemic foci of ZCL in neighbouring countries were reported in Saudi Arabia (Morsy and Shoura, 1976, Sebai and Morsy, 1976; Morsy, 1988). On the same time, the report of ACL in Sinai (Shehata et al, 2009) and the endemicity of the ACL was reported in Saudi Arabia (Morsy et al, 1991b) Jordan (Saliba et al, 1997) and its presence on the Egyptian Eastern border with Palestine (Azmi et al, 2010; 2012), South border with the Sudan (Babiker et al, 2014) and the western border with Libya (Fathy et al, 2009; El-Badry et al, 2016) as well as the abundance of specific vector $P$. sergenti (Lane, 1986, 1993) in Egypt and Arab countries (El Sawaf et al, 2016; Sawalha et al, 2003 ; 2017) is another risk factor.

\section{Conclusion}

Based on the outcome data: 1- Indeed, there was distinct risk of acquiring zoonoticcutaneous leishmainsis among the manual personnel in remote area in the north Sinai Governorate. 2- Fortunately, the disease was presented as a small skin lesion(s) with minimal risk of complications that limited to secondary infection or residual black scar, mainly in lower limb and rarly on face. 3More frequent risk factors as reported, might be naturally present such as, the mountains, the desert vegetations, sandy ground and presence of wild rodents as a main reservoir host, or manmade induced such as primitive building with wall containing cracks, not paved floor, ceiling made of bumbo (arbor), places of the sand fly aggregate especially water cycles. 4- At high risk group, susceptible persons coming from ZCL-free zones to the endemic area, with lack of endemicity knowledge, inadherence to standard protective measures such as applying insect repellent or using the bed nets.

ZCL should be considered as a cause of skin lesion in relevant target groups coming from Northern Sinai. The infection was most common in the younger age group and clusters in summer months. Rodent's reservoir hosts common, bathroom area commonest breeding site for sand flies aggregate.

ZCL was constantly associated with: Residence in desert or semi-desert areas, where naturally planted with certain types of desert vegetation. Primitive building design associ 
ated with decreased number of floors, crevices /cracks in the unplastered racticed wall, and ceiling constructed by arbor which considered a suitable shelter for rodents, as well as the lack of the health education. Monotherapy with Na-stibogluconate was the superior to oral fluconazole, while both laser and cryotherapy proved best among former medications.

\section{Recommendations}

The Public Health and Veterinay-Medical Authorities must take into consideration that the ZCL is more or less endemic in all the countries bordering Egypt. The quantification and the containment of the elicited risk factor for zoonotic cutaneous leishmaniasis in the light of study results are major challenges and should be considered by health policy maker and public health professionals, in order to evaluate (CL) burden and to highlight priority actions for the disease control. Thus, it was recommended to: 1 - To do massive rodent control at least in and outdoors: by setting short-term control plans. 2Vector control: combating sand fly-vectors by all the possible environmental friend means in and around human vacinity. 3Early detection and pro-per treatment of cases, lesions must be covered with proper dressing to avoid autoinfection and infection to vectors,. 4- Decrease human-sand fly contact by simple measures as screening of widows, avoiding sleeping out-door especially in near to the bathroom, applying repellent regularly, wearing long sleeved shirts and pants. 5-Modifying natural environment in trial to eradicate the existing desert vegetation and replaced it with an asphalt, also taking into consideration, the construction of the sanitary building as high floor design, plastering wall, tiling the floor and concreting the ceiling. 6- To conduct active public health surveillance including the statistical spatial and space-time analysis for feasible planning of successful control strategy. 7To identify Leishmania species of anthroponotic cutaneous leishmaniasis (ACL) in Sinai, since its specific vector by typing and/or characterization proved to be present, and 8- Health education for the immigrant workers and employees to Sinai and the endemic neihbouring countries.

\section{References}

AbdeIlatif, MZM, El-Mabrouk, K, Ewis, AA, 2012: Cutaneous Leishmaniasis infection in Aljabal Al-gharbi, Libya, an epidemiological study. Korean J. Parasitol. 50, 4:127-44.

Abdel-Motagaly, AMEl, Morsy, TA, 2016:

Zoonotic cutaneous leishmaniasis three years fever hospital records: With general discussion. J. Egypt. Soc. Parasitol. 46, 3:527-32.

AbduIsadah, AR, 2011: Cutaneous leishmaniasis at Wasit Governorate. Baghd. Sci. 8, 2:286-8. Ahmed, SM, Abou Faddan, HH, 2013: Cutaneous leishmaniasis In Gharyan, Libya: A case-control study. Life Sci. J. 10, 1:826-34.

Al-Jawabreh, A, Dumaidi, K, Ereqat, S, AlJawabreh, H, Nasereddin, A, et al, 2016: Molecular epidemiology of human cutaneous leishmaniasis in Jericho and its vicinity in Palestine from 1994 to 2015. Infect. Genet. Evol. 2016 Jun 5. pii: S1567-1348(16)30231-3

Amer, MA, Morsy, TA, 1995: Misir' da kutan oz Laysmanyazis Epide-miyolojisi: (Epidemiology of cutaneous leishmaniasis in Egypt). Turk Dermat, Deri Hastalklari Ve Frengi Arsivi, 29, 2:84-7, Turkey

Antinori, S, Giamelli, E, Calattini, S, Longhi, E, Gramiccia, M, et al, 2005: Cutaneous leishmaniasis: an increasing threat for treavellers. Clin. Microbiol. Infect.11, 5:343-6.

Azmi, K, Nasereddin, A, Ereqat, S, Schonian, G, Abdeen, Z, 2010: Identification of Old World Leishmania species by PCR-RFLP of the 7 spliced leader RNA gene and reverse dot blot assay. Trop. Med. Int. Hlth. 15:872-80.

Azmi, K, Schönian, G, Nasereddin, A, Schnur, LF, Sawa-lha, S, et al, 2012: Epidemiological and clinical features of cutaneous leishmaniases in Jenin District, Palestine, including characterization of the causative agents in the clinical samples. Trans. Roy. Soc. Trop. Med. Hyg. 106, 9:554-62.

Babiker, AM, Ravagnan, S, Fusaro, A, Hassan, MM, Bakheit, SM, et al, 2014: Concomitant infection with Leishmania donovani and $L$. major in single ulcers of cutaneous leishmaniasis patients from Sudan. J. Trop. Med. 170859. doi: 10.1155/2014/170859.

Bassili, WR, Morsy, TA, Michael, SA, 1983: 
Specific and sensitivity of indirect haemagglutination tests in patients with cutaneous leishmaniasis. J. Egypt. Soc. Parasitol. 13:291-5.

Ben-Shimol, S, Sagi, O, Horev, A, Avni, YS, Ziv, M, et al, 2016: Cutaneous leishmaniasis caused by Leishmania infantum in Southern Israel. Acta Parasitol. 61, 4:855-8.

Bousslimi, N, Aoun, K, Ben-Abda, I, Ben-Alaya-Bouafif, N, Raouane, M, 2010: Epidemiologic and clinical features of cutaneous leishmaniasis in southeastern Tunisia. Am. J. Trop.

Med. Hyg. 83:1034-9.

Bruce, FE, John, DE, 2004: A Textbook on Public Health and Veterinary Problems Caused by Arthropods: Medical Entomology.

Das, ML, Singh, SP, Vanlerberghe, V, Rijal, S, Rai, M, 2007: Population preference of net texture prior to bed net trial in kala-azar- endemic areas. PLoS Negl. Trop. Dis. 1:e100.

Dawoud, HA, 2004: Identification of cutaneous leishmaniasis in Egypt by hybridization of PCR amplified mini-exon repeats. J. Egypt. Soc. Parasitol. 34, 3:881-92.

Eisa, S, Abdel-Ati, A, Ebrahim, M, Eid, M, Abd El-Samad, E, Sayed Hussin, S, 2014: Bridging the gap between increasing knowledge and decreasing resources: Chenopodium quinoa as a New Non-Traditional Crop in Egypt. Tropentag, September 17-19, 2014, Prague, Czech Republic

El-Badry, AA, El-Dwibe, H, Basyoni, MM, Al-Antably, A S, Al-Bashier, WA, 2016: Molecular prevalence and estima-ted risk of cutaneous leishmaniasis in Libya. J. Microbiol. Immunol. Infect. Jan 12. pii: S1684-1182(16)00005-0. doi: 10.1016/j.jmii.2015.12.004.

El Gibali, MR, El Mansouri, H, 1979: Pyrimethamine in prophylaxis and treatment of cutaneous leishmaniasis. Armed Forces Med. J. 11:

El Sawaf, BM, Shoukry, A, el Said, S, Lane, R P, Kenawy, MA, et al, 1987: A brief report on sandflies in southern Sinai, Egypt. J. Egypt. Soc. Parasitol. 17, 1: 413-4.

El Sawaf, BM, Kassem, HA, Mogalli, NM, El Hossary, SS, Ramadan, NF, 2016: Current knowledge of sand fly fauna (Diptera: Psychodidae) of northwestern Yemen and how it relates to leishmaniasis transmission. Acta Trop. 162:11-9 Fahmy, AR, Samy, AM, Doha, SA, Shehata, MG, 2009: Preliminary field investigations on phlebotomine sandflies (Diptera: Psychodidae) from a recent cutaneous leishmaniasis focus in
Northern-Sinai, Egypt. Egypt. Acad. J. Biol. Sci. 2:9-15

Faris, R, Feinsod, FM, Morsy, TA, ElMissiry, AG, Gabal, MS, et al, 1988: Human cutaneous leishmaniasis in two communities in Eastern Sinai, Egypt. Eur. J. Epidemiol. 4, 1:45-8.

Faris, R, Morsy, TA, Feinsod, FM, Gabal, S, EIMissiry, AG, et al, 1986: Population based studies of cutaneous leishmaniasis in North Sinai. Ann. Meet. Am. Soc. Trop. Med. Hyg. Denver, Co., USA.

Fathy, FM, El-Kasah, F, El-Ahwal, AE, 2009: Emerging cutaneous leishmaniasis in Sirte-Libya: Epidemiology, recognition and management. J. Egypt. Soc. Parasitol. 39, 3:881-905.

Feliciangeli, MD, Delgado, O, Suarez, B, Bravo, A, 2006: Leishmania and sand flies: proximity to woodland as a risk factor for infection in a rural focus of visceral leishmaniasis in west central Venezuela. Trop. Med. Int. Hlth. 12, 2:178591.

Fuentes-Bazan, S, Mansion, G, Borsch, T, 2012: Towards a species level tree of the globally diverse genus Chenopodium (Chenopodiaceae). Mol. Phyl. Evol. 62:359-74.

Hamadto, HA, El Fakahany, AF, Morsy, TA, Farrag, A, Abdel Maksoud, KhM, 2003: Reevaluation of zoonotic cutaneous leishmaniasis status in North Sinai Governorate, Egypt. J. Egypt. Soc. Parasitol. 33, 3:687-94.

Hamadto, HA, AI-Fakahany, AF, Farrag, A B, Abdel-Maksoud, MK, Morsy, TA, 2007: Zoonotic cutaneous leishmaniasis: reservoir host and insect vector in north Sinai, Egypt. 1. Egypt. Soc. Parasitol. 37, 3:843-50.

Hanafi, HA, Beavers, GM, Dykstra, EA, 2001: New record of Phlebotomus sergenti, the vector of Leishmania tropica, in the southern Nile valley of Egypt. J. Am. Mosq. Control Assoc. 17, 4:272-4.

Karimkhani, C, Wanga, V, Coffeng, LE, Naghavi, P, Dellavalle, RP, et al, 2016: Global burden of cutaneous leishmaniasis: A cross-sectional analysis from the global burden of disease study 2013. Lancet Infect. Dis. 16, 5:584-91

Killick Kendrick, R, 1999: The biology and control of Phlebotomine sand flies. Clin. Dermatol. 17:279-89.

Klemper, MS, Unnasch, TR, Linden, TH, 2007: Taking a bite out of vector-transmitted infectious diseases. N. Engl. J. Med. 356, 25:67-9. Lane, RP, 1986: The sandflies of Egypt (Dipte- 
ra: Phlebotominae). Bull. Br. Mus. Nat. Hist. (Entomol.) 52:1-35.

Lane, R.P, 1993: Sand flies (phlebotomine). In: Medical Insects and Arachnids: RP, Lane, and RW, Crosskey (Eds.), Chapman and Hall, London, United Kingdom.

Lewis, DJ, 1971: Plebotomid Sandflies. Bull WHO 44, 4:535-51.

Mansour, NS, Youssef, FG, Mohareb, EW, Dees, WH, Karuru, ER, 1987: Cutaneous leishmaniasis in north Sinai. Trans. R. Soc. Trop. Med. Hyg. 81, 5:747.

Merdan, AI, Morsy, TA, Shoukry, A, Wahba, MM, 1992: Two successive years studies on $P h$ lebotomus papatasi in North Sinai Governorate, Egypt. J. Egypt. Soc. Parasitol. 22, 1:91-100.

Mohammadi, KA, Alhussainy, NH, 2014: Incidence of leishmaniasis in Al Baha Province, Saudi Arabia: past and present situation (observational and descriptive study). J. Egypt. Soc. Parasitol. 44, 3:591-6.

Moosa-Kazemi, SH, Yaghoobi-Ershadir, MR. Akhavan, AA, Abdoli, H, Zahraei, AR, 2007: Deltamethrin-impregnated bed nets and curtains in an anthroponotic cutaneous leishmaniasis control program in northeastern Iran. Ann. Saudi Med. 27:6-12.

Morsy, TA, 1983: Cutaneous leishmaniasis in Egypt. J. Egypt. Soc. Parasitol. 13, 2:597-611. Morsy, TA, 1996: Cutaneous leishmaniasis in Egypt, (Review and comment). J. Egypt. Soc. Pa-rasitol. 26:105-30.

Morsy, TA, 1988: WHO/EMRO/ST/Consultant for Leishmaniasis Control in Saudi Arabia.

Morsy, TA, 2013: Cutaneous leishmaniasis predisposing to human skin cancer: Forty years local and regional studies. J. Egypt. Soc. Parasitol. 43, 3:629-48.

Morsy, TA, Shoura, MI, 1976: Some aspects of cutaneous leishmaniasis in Riyadh. Saudi Arabia. J. Trop. Med. Hyg. 79, 6:137-9.

Morsy, TA, el Ajlouny, AK, 1984: Cutaneous leishmaniasis in Jordan. J. Egypt. Soc. Parasitol. 14, 1:257-64.

Morsy, TA, Bassili, WR, Fayad, ME, El Okbi, LM, Saleh, MSM, 1987a: Rodents in relation to cutaneous leishmaniasis in North Sinai Governorate, Egypt. J. Egypt. Soc. Parasitol. 17, 2: 427-38.

Morsy, TA, Shoukry, A, Schnur, LF, Sulitzeanu, A, 1987b: Gerbillus pyramidum is a host of Leishmania major in the Sinai Peninsula. Ann. Trop. Med. Parasitol. 81, 6:741-2
Morsy, TA, Schnur, LF, Feinsod, FM, Salem, AM, Wahba, MM, et al, 1987c: Natural infections of Leishmania major in domestic dogs from Alexandria, Egypt. Am. J. Trop. Med. Hyg. 37, 1:49-52.

Morsy, TA, Shoukry, A, Mazyad, SAM, Makled, KA, 1998: The effect of the volatile oils of Chenopodium ambrosioides \& Thymus vulgaris against the larvae of Lucilia sericata (Meigen). J. Egypt. Soc. Parasitol. 28, 2:503-10.

Morsy, TA, Abdel Rahman, EG, Ahmed, M M, 1989: Treatment of cutaneous leishmaniasis with pentostam or cryosurgery. J. Egypt. Soc. Parasitol.19, 2:533-43.

Morsy, TA, El-Missiry, AG, Kamel, A, Fayad ME, el-Sharkawy, I, 1990: Distribution of $P h$ lebotomus species in the Nile Delta, Egypt. J. Egypt. Soc. Parasitol. 20, 2:589-97.

Morsy, TA, Salama, MMI, Saleh, MS, 1991: Leishmania major and Meriones crassus in North Sinai Governorate, Egypt. J. Egypt. Soc. Parasitol. 21, 2:337-42.

Morsy, TA, Merdan, AI, Shoukry, A, Wahba, MM, 1992: Isonezyme characterization of Leishmania major isolated from three patients in North Sinai, Egypt. J. Trop. Med. 2, 5:61-4

Morsy, TA, El Kady, GA, Salama, MMI, Habib, KMS, Ghareb, MA, 1993: Leishmania major in Meriones sacramenti Thomas 1992 in Nakhl, North Sinai, Egypt. J. Egypt. Soc. Parasitol. 23, 2:371-9.

Morsy, TA, el Shazly, AM, el Kady, GA, Sabry, AH, Handousa, AA, et al, 1994: NaturalLeishmania infections in two stray dogs and two Gerbillus pyramidum in Dakahlia Governorate, Egypt. J. Egypt. Soc. Parasitol. 24, 2:383-94

Morsy, TA, Naser, AM, El Gibali, MR, Anwar, AM, El Said, AM, 1995: Studies on zoonotic cutaneous leishmaniasis among a group of temporary workers in North Sinai Governorate, Egypt. J. Egypt. Soc. Parasitol. 25, 1:99-106.

Morsy, TA, Sabry, AA, Rifaat, MMA, Wahba, MM, 1996: Psammomys obesus Cretzchmar, 1828 and zoonotic cutaneous leishmaniasis in Sinai Peninsula, Egypt. J. Egypt. Soc. Parasitol. 26, 2:375-81.

Morsy, TA, Ibrahim, BB, Lashin, AH, 1997: Leishmania major in an Egyptian patient manifested as diffuse cutaneous leishmaniasis. J. Egypt. Soc. Parasitol. 27, 1:205-10.

Morsy, TA, Saleh, WA, Ismail, MAM, 2002: The red fox Vulpes $O$. aegyptica, a new host of 
Leishmania major in Sinai Peninsula. J. Egypt. Soc. Parasitol. 32, 3:737-43.

Osbern, DJ, Helmy, I, 1980: Fieldiana Zoology: The Contemporary Land Mammals of Egypt (Including Sinai). Published by Reid Museum of Natural History: New Series no. 5, Publication 1309.

Peterson, AT, 2008: Biogeography of diseases: a framwork for analysis. Natur. Wiss 95:483-91.

Postigo, JA, 2010: Leishmaniasis in the World Health Organization Eastern Mediterranean Region. Int. J. Antimicrob. Agents 36, 1:S62-5.

Ranjan A, Sur D, Singh, A, 2005: Risk factors for Indian Kala-azar. Am. J. Trop. Med. Hyg. 73:74-8.

Saleh, AMA, Labib, NA, Abdel-Fattah, MS, Al-Attar, MBF, Morsy, TA, 2015: Sand-fly Phlebotomus papatasi (Phlebotominae): A general review with special reference to zoonotic cutaneous leishmaniasis in Egypt. J. Egypt. Soc. Parasitol. 45, 3:525-41

Saliba, Ek, Saleh, N, Oumeish, OY, Khoury, S, Bisharat, Z, et al, 1997: The endemicity of Leishmania tropica (zymodeme MON-137) in the Eira-Yarqa area of Salt District, Jordan. Ann. Trap. Med. Parasitol. 91, 5:453-9.

Sawalha, SS, Shayeh, MS, Khanfar, HM, Warburg, A, Warbura, ZA, 2003: Phlehotomine sand flies (Diptera: Psychodidae) of the Palestinian West Bank: potential vectors of leishmaniais. J. Med. Entomol. 40: 321-8.

Sawalha, SS, Ramlawi, A, Sansur, RM, Salem, IM, Amr, ZS, 2017: Diversity, ecology, and seasonality of sand flies (Diptera: Psychodiidae) of the Jenin District (Palestinian Territories). J. Vector Ecol. 42, 1:120-9.

Schnur LF, Nasereddin A, Eisenberger CL, Jaffe CL, El Fari M, et al, 2004: Multifarious characterization of Leishmania tropica from a Judean desert focus, exposing intraspecific diversity and incriminating Phlebotomus sergenti as its vector. Am. J. Trop. Med. Hyg. 70:364-72.

Sebai, ZA, Morsy, TA, 1976: Cutaneous leishmaniasis in Bisha Town, Saudi Arabia. J. Trop. Med. Hyg. 79, 4:89-91.

Shehata MG, Samy AM, Doha SA, Fahmy AR, Kaldas RM et al, 2009: First report of Leishmania tropica from a classical focus of $L$. major in North-Sinai, Egypt. Am. J. Trop. Med. Hyg. 81, 2:213-8.

Shehata, MG, Samy, AM, Doha, SA, Fahmy, AR, Kaldas, RM, et al, 2009: First report of Leishmania tropica from a classical focus of L. major in North-Sinai, Egypt. Am. J. Trop. Med. Hyg, 81:213-8

Voisin, C, Bianchi, F, Parent, D, Evrard, L, 2011: Leishmaniasis of the lip: Report of a case. Bull. Group Int. Rech. Sci. Stomatol. Odontol. 50, 1:6-10.

Wahab, MM, Schnur, LE, Morsy, TA, Merdan, A, 1990: The characterization of Leishmania major from Phlebotomus papatasi. J. Trop. Med. Hyg. 84:758-9.

WHO, 2013: Leishmaniasis: Burden of Disease: World Health Organization; Geneva, Switzerland. 